\title{
Coating Mechanisms of Single-walled Carbon Nanotube by Linear Polyether Surfactants: Insights from Computer Simulations
}

\author{
Edita Sarukhanyan $^{a, b}$, Giuseppe Milano ${ }^{b, c}$, Danilo Roccatano $^{a^{*}}$ \\ ${ }^{a}$ Jacobs University Bremen, Campus Ring 1, D-28759 Bremen, Germany \\ ${ }^{\mathrm{b}}$ Dipartimento di Chimica e Biologia and NANOMATES, Research Centre for NANOMAterials \\ and nanoTEchnology at Università di Salerno, I-84084 via Ponte don Melillo Fisciano (SA), Italy \\ ${ }^{\mathrm{c}}$ IMAST Scarl-Technological District in Polymer and Composite Engineering, P.le Fermi 1, 80055 \\ Portici (NA), Italy
}

AUTHOR EMAIL ADDRESS. d.roccatano@jacobs-university.de

\section{CORRESPONDING AUTHOR:}

Prof. Dr. Danilo Roccatano. Jacobs University Bremen, Campus Ring 1, D-28759, Bremen, Germany. Fax: +49 421 200-3249, Tel: +49 421 200-3144, E-mail: d.roccatano@jacobsuniversity.de. 


\begin{abstract}
.
The non-covalent coating of carbon-based nanomaterials, such as carbon nanotubes, has important applications in nanotechnology and nanomedicine. The molecular modeling of this process can clarify its mechanism and provide a tool for the design of novel materials. In this paper, the coating mechanism of single-walled carbon nanotubes (SWCNT) in aqueous solutions by 1,2 dimethoxyethane oxide (DME), 1,2 - dimethoxypropane oxide (DMP), polyethylene oxide (PEO), polypropylene oxide (PPO) pentamers, and L64 triblock copolymer chains have been studied using molecular dynamics (MD) simulations. The results suggest a preferential binding to the SWCNT surface of the DMP molecules with respect to DME mainly driven by their difference in hydrophobicy. For the longer pentamers, it depends by the chain conformation. PPO isomers with radius of gyration larger than PEO pentamers bind more tightly than those with more compact conformation. In the case, of the L64 triblock copolymer, the coating of the SWCNT surface produce a shell of PPO blocks with the PEO chains protruding into bulk water as expected from the so-called non-wrapping binding mechanism of SWCNT. In addition, polymer coating, qualitative agreement with experimental evidences on the poor capability of the L64 to disperse SWCNT, do not prevent the formation of CNT aggregates.
\end{abstract}

KEYWORDS. Carbon nanotube aggregation, 1,2-dimethoxyethane oxide, 1,2-dimethoxypropane oxide, polyethylene oxide, polypropylene oxide, Pluronics. 


\section{INTRODUCTION.}

Carbon nanotubes (CNT) show a peculiar ability to penetrate biological membrane and cumulate in the cell. ${ }^{1}$ This characteristic, together with other properties of this material, offers interesting alternatives for the development of novel delivery systems in therapy and in diagnostics. ${ }^{2-3}$ Nevertheless, this potential use is mainly limited by their poor solubility in water: newly synthesized single-walled carbon nanotubes aggregate in bundles that are actually difficult to separate. ${ }^{4}$ Different approaches, based on the CNT surface modification, have been proposed to overcome this problem ${ }^{5-6}$. However, for possible biomedical applications, CNT derivatives should not only improve the CNT solubility, but also sustain biocompatibility and low toxicity. CNTs surface modifications can be accomplished by covalently bound functional groups ${ }^{7}$ and/or by noncovalently coating the surface with surfactants or, in general, amphipathic molecules like lipids and polymers. ${ }^{8}$ In particular, block copolymers have been successfully used for modifying the solution behaviour of SWCNTs and multi-walled CNTs (MWCNT). These amphipathic polymers are able to disperse the nanotubes in different media in order to reduce their assembly into bundles. ${ }^{9-11}$ Unfortunately, the molecular details of this process are not yet clarified. In a recent study on the adsorption of block copolymers to SWCNT's and MWCNT's, a so-called non-wrapping mechanism of coating the nanotube surface ${ }^{12}$ has been proposed. In this model, the more hydrophobic PPO blocks are adsorbed onto the nanotube surface, while the more hydrophilic PEO units remain extended into the aqueous solution, providing a steric repulsion that determines the dispersion of the CNTs. In another study, Granite et al. ${ }^{13}$ investigated interactions between block copolymers and SWCNTs in aqueous solutions using the small-angle neutron scattering technique. In this study, two models of polymers-SWCNT interaction have been suggested. In the first, the core-chain model, polymer chains are adsorbed onto the small bundle (the core) surface and there is no difference between PEO and PPO components. In the second more detailed one, the core-shell-chains model, the surface of the core (SWCNT bundle) is in contact with the shell (the region composed by the 
PPO units of the polymer) and the PEO units (the chains part of the model) extend in the solvent phase. Frise and coworkers ${ }^{14}$ have also studied the interaction between the block copolymer F127 and CNT in aqueous dispersion by pulsed-field gradient (PFG) ${ }^{1} \mathrm{H}$ NMR spectroscopy. Their study confirmed the previous studies by showing that the polymer binds CNTs by its central hydrophobic block, while the two hydrophilic PEO terminal parts extend into water phase.

Different theoretical studies on the interaction of different polymers and biopolymers with SWCNT are present in the literature. ${ }^{15} \mathrm{MD}$ simulation studies have been performed to investigate the coating mechanism of the nanotube by polymeric chains but few of them have extensively study the interaction of polyethers with SWCNT. Nativ-Roth et al. have used short (1 ns) MD simulation of the Pluronics F88 to a SWCNT $(10,10)$ to support their experimental results on the preferential binding of the nanotube surface. ${ }^{12}$ The simulation has qualitatively shown a tight binding of the PPO block to the nanotube and the presence of extended chain configurations of the PEO blocks in the solvent. ${ }^{12}$ The effect of the SWCNT diameter on the interaction with polymers have been recently address using MD simulations by Yang et al. ${ }^{16}$ They have studied the interaction of polystyrene (PS), poly(phenylacetylene) (PPA), poly(p-phenylvinilene) (PPV), and poly(mphenylenevinilene-co-2.5-dioctyloxy-p-phenylenevinilene) (PmPV) with "Zig-zag" type SWCNTs of length of $10.38 \mathrm{~nm}$ and diameters ranging from 0.39 to $5.36 \mathrm{~nm}$. All the polymers showed a tendency to coat the SWCNT surface. However, the strength of the bindings was found to be very dependent on the structure of polymer. In particular, PPV and PmPV polymers, consisting of monomers with aromatic groups in their backbone, tend to bind the SWCNT surface stronger than PS and PPA, containing the aromatic groups in their side chains.

In this paper, we report an extensive theoretical study, based on atomistic MD simulations, of different types of SWCNT in the presence of linear polyether surfactants. We have used recent models of polyether polymers specifically optimize to accurately reproduce the physical chemistry properties in water solution. ${ }^{17-18}$ The paper is divided in three parts. In the first part, the coating 
mechanism of SWCNTs of different diameters by 1,2 -dimethoxyethane (DME), 1,2 dimethoxypropane oxide (DMP), the smallest oligomers ( $\mathrm{n}=1$ in the Scheme 1S) of polyethylene oxide (PEO) and polypropylene oxide (PPO), respectively was analyzed. Since DME and DMP can be considered the smallest units for PEO and PPO polymers, respectively, the aim of this study was to provide a detailed model of differential binding interactions of the two units monomers with SWCNT's. Nanotubes of different diameter and chirality have been used to understand the effect of the surface area and curvature on the polymer adsorption. In fact the high surface/volume ratio present in SWCNTs may have a strongly affect on the adsorption capability of polymeric materials. ${ }^{15}$ In the second part of the study the effect of the polymer length and tacticity on the absorption of the SWCNT $(5,5)$ was investigated using pentamers $(n=5$ in the Scheme $1 \mathrm{~S})$ of PEO $\left(\mathrm{PEO}_{5}\right)$ and of different isomers of PPO $\left(\mathrm{PPO}_{5}\right)$. Finally, in the last part, the absorption of the small triblock copolymer L64 (see Scheme 1S) on a single and a SWCNT $(5,5)$ bundle formation was studied. The aim of this last part was to analyze whether the absorption mechanism obtained from our model systems agrees with the available experimental and theoretical data.

\section{COMPUTATIONAL METHODS.}

\section{Force Field parameters.}

Single-walled carbon nanotubes with chiral indices $m=10, n=5$ (chiral), $m=7, n=7$ (arm-chair), $m=5$, $n=5$ (arm-chair) and $m=5, n=0$ (zig-zag) and length of $3 \mathrm{~nm}$ have been used to perform simulations. The SWCNTs used in these simulations are without explicit hydrogen atoms at the edges. Since nanotubes, used in experimental work are much longer $\left(\sim 500 \mathrm{~nm}^{13}\right)$ than our models, the charges at both edges of SWCNT should not affect the interactions of the polymers far away from the edges. In Table 1, the list of SWCNTs used in this study with the corresponding diameters and chiral indices are reported.

The SWCNT force field is based on the model proposed by Walther et al. ${ }^{19}$ The carbon atoms were considered uncharged. The Lennard-Jones (LJ) parameters $\varepsilon_{c c}, \sigma_{c c}$ for carbon-carbon interaction of 
nanotube was chosen to be $0.4396 \mathrm{kJmol}^{-1}$ and $0.3851 \mathrm{~nm},{ }^{19}$ respectively. LJ parameters of interaction between the carbon of the nanotube and oxygen of the water were set to $\varepsilon_{\mathrm{co}}=0.392$ $\mathrm{kJmol}^{-1}$ and $\sigma_{\mathrm{co}}=0.319 \mathrm{~nm},{ }^{20}$ respectively. The mixed $\mathrm{LJ}$ parameters for the interaction of the SWCNT carbon with other atoms were obtained using the combination rules: ${ }^{21}$

$$
C_{i j}^{(6)}=\sqrt{C_{i i}^{(6)} C_{j j}^{(12)}} \quad C_{i j}^{(12)}=\sqrt{C_{i i}^{(12)} C_{j j}^{(12)}}
$$

where $C_{i i}^{(6)}, C_{j j}^{(12)}$ and $C_{i j}^{(6)}$ are the LJ coefficients for the interaction between the particles of type $i$ with the particle of type $j$ and $C_{i i}^{(6)}=4 \varepsilon_{i i} \sigma_{i i}^{6}$ and $C_{j j}^{(12)}=4 \varepsilon_{i i} \sigma_{i i}^{12}$, respectively. In this work, we have assumed that the LJ parameters of the carbon atoms are the same in all the SWCNT i.e. they are not significant affected by the change of the electronic properties of the carbon atoms due to the CNT curvature.

The models of DME, DMP, $\mathrm{PPO}_{5}, \mathrm{PPO}_{5}$ and Pluronics L64 (see Scheme 1S) were developed in our research group and the details of the models have been recently published. ${ }^{17-18}$ All polymers used in this study have methyl groups as terminal groups. For the $\mathrm{PPO}_{5}$, five different isomers have been studied. The isomers are indicated with the $\mathrm{R} / \mathrm{S}$ descriptors of each stereo center assigned using the Cahn-Ingold-Prelog system. For the L64 polymer, we have used the same atactic sequence as in out previous paper. ${ }^{18}$ The atactic sequence (PEO 13 - $\underline{\text { RRSRRRRRSRSRRRSSRRSSRSSRSRRRRR- }}$ $\mathrm{PEO}_{13}$ ) contains the same five isomers (four underlined and in one in bold characters) chosen for the $\mathrm{PPO}_{5}$ chains.

Table 1: Chiral indices of SWCNT and corresponding diameters.

\begin{tabular}{ccc}
\hline Chiral indices $(\boldsymbol{m}, \boldsymbol{n})$ of SWCNT & Geometric & External van der Waals \\
& Diameter, $\mathbf{n m}$ & Surface Area*, $\mathbf{n m}^{\mathbf{2}}$ \\
\hline$(10,5)$ & 1.04 & 18.00 \\
& $\mathbf{6}$ &
\end{tabular}


0.95

0.68

0.39
17.22

14.66

11.96

* A diameter extended by $2 \sqrt[6]{2} \sigma_{C O}$ was used for the estimation.

MD Simulations. The systems were prepared centering the SWCNTs in a box of suitable size (see Table 2). The empty space was then filled with the solvent molecules (DME/DMP and/or water) by geometrically stacking equilibrated boxes of the pure solvents. For the simulation with PEO, PPO pentamers and the L64 triblock copolymer, the chains have been initially regularly placed inside in a box with the nanotube at the center and then filled with water molecules as described before. A summary of the simulated systems is reported in Table 2. All the simulations were performed at constant temperature and pressure (NPT conditions) using periodic boundary conditions. The integration time step was chosen to be $2 \mathrm{fs}$. The temperature and pressure were maintained to the reference values $(\mathrm{T}=298 \mathrm{~K}, \mathrm{P}=1$ bar) using the Berendsen thermostat and barostat with coupling time constant of $\tau_{\mathrm{T}}=0.1 \mathrm{ps}$ for temperature and $\tau_{\mathrm{p}}=0.5 \mathrm{ps}$ for pressure, respectively. The Extended Simple Point Charge (SPC/E) model was used as water model. ${ }^{22}$ The SETTLE $^{23}$ algorithm was used for the water molecules. The electrostatic interactions were calculated by using the Particle Mesh Ewalds (PME) method. ${ }^{24}$ For the long-range interactions, a grid spacing of $0.12 \mathrm{~nm}$ combined with a fourth-order B-spline interpolation were used to compute the potential and forces between grid points. A pair-list for non-bonded interactions within the cutoff of $1 \mathrm{~nm}$ was used and updated at every 10 time-steps. In the simulations, all-bonds were constraint using the LINCS algorithm. ${ }^{25}$

All the systems have been energy minimized using the steepest descent algorithm for at the least 1000 steps to remove the possible clashing. After energy minimization, initial velocities obtained from Maxwell-Boltzmann velocity distributions at $298 \mathrm{~K}$ were assigned to all atoms. All systems were initially equilibrated for $100 \mathrm{ps}$ with position restraints on the solute atoms for the relaxation 
of water molecules. Hence, the simulation runs without position restraints and of different length (see Table 2) have been performed.

Table 2: Summary of the simulated systems. In the system name the notation $\mathrm{qS}(n, m)$ indicate a number q of SWCNT with chirality index $(n, m)$.

\begin{tabular}{|c|c|c|c|c|c|c|}
\hline System & $\begin{array}{l}\text { Simul. } \\
\text { length, } \\
\text { ns }\end{array}$ & $\begin{array}{l}\text { Box side } \\
\text { size, } \text { nm }^{3}\end{array}$ & $\begin{array}{l}\text { Water } \\
\text { mol. }\end{array}$ & $\begin{array}{l}\text { DME/ } \\
\text { PEO } \\
\text { chains }\end{array}$ & $\begin{array}{l}\text { DMP/ } \\
\text { PPO } \\
\text { chains }\end{array}$ & $\begin{array}{l}\text { L64 } \\
\text { chains }\end{array}$ \\
\hline S(10,5)DME:DMP & 25 & 5.5 & 5196 & 23 & 24 & - \\
\hline S(7,7)DME:DMP & 25 & 5.5 & 5202 & 23 & 24 & - \\
\hline S(5,5)DME:DMP & 25 & 5.5 & 5196 & 23 & 24 & - \\
\hline S(5,0)DME:DMP & 25 & 5.5 & 5212 & 23 & 24 & - \\
\hline $\mathrm{S}(5,5) \mathrm{EO}_{5}$ & 75 & 10.0 & 32864 & 15 & - & - \\
\hline $\mathrm{S}(5,5) \mathrm{PO}_{5}(R R R R R)$ & 75 & 10.0 & 32773 & - & 15 & - \\
\hline $\mathrm{S}(5,5) \mathrm{PO}_{5}(R S R S R)$ & 75 & 10.0 & 32773 & - & 15 & - \\
\hline $\mathrm{S}(5,5) \mathrm{EO}_{5}: \mathrm{PO}_{5}(R R R R R)$ & 75 & 10.0 & 32846 & 7 & 7 & - \\
\hline $\mathrm{S}(5,5) \mathrm{EO}_{5}: \mathrm{PO}_{5}(R S R S R)$ & 75 & 10.0 & 32846 & 7 & 7 & - \\
\hline $\mathrm{S}(5,5) \mathrm{EO}_{5}: \mathrm{PO}_{5}(R R S R R)$ & 75 & 10.0 & 32846 & 7 & 7 & - \\
\hline $\mathrm{S}(5,5) \mathrm{EO}_{5}: \mathrm{PO}_{5}(R R S R R)$ & 75 & 10.0 & 32846 & 7 & 7 & - \\
\hline $\mathrm{S}(5,5) \mathrm{EO}_{5}: \mathrm{PO}_{5}(R S S R S)$ & 75 & 10.0 & 32846 & 7 & 7 & - \\
\hline $\mathrm{S}(5,5) \mathrm{L} 64$ & 75 & 10.0 & 32192 & - & - & 6 \\
\hline $3 \mathrm{~S}(5,5)$ & 80 & 4.5 & 2751 & - & - & - \\
\hline $3 \mathrm{~S}(5,5) \mathrm{L} 64$ & 100 & 10.0 & 31716 & - & - & 12 \\
\hline
\end{tabular}

Analysis of the Trajectories. The distribution of the solvent and surfactants around the SWCNT was analyzed using cylindrical radial distribution function (cRDF), cylindrical cumulative coordination number, and density profile along the nanotube axial direction.

All the distributions were calculated using cylindrical coordinate system $(r, \varphi, z)$, with origin at the center of mass $(\mathrm{CoM})$ of the SWCNT. Here $r$ is a radial distance from the axis of the nanotube, $\varphi$ azimutal angle; $\mathrm{z}$ is the position along the axis of the nanotube (see Figure 1).

The interior region of the nanotube is defined as the volume $\mathrm{V}_{0}$ of a cylinder of height $h$ given by the nanotube length, and with a base of diameter $d_{0}$ equal to $V_{0}=\frac{\pi}{4} d_{0}^{2} h$. The volume of the nanotube shell is defined by the volume of the hollow cylinder of diameter $d$ around the SWCNT 
external surface equal to $V_{h c}=\frac{\pi}{4} d^{2} h-V_{0}$. Solute molecule is considered to be inside the nanotube or in the shell region if its center of mass is located inside the one of the above-defined volumes.

The cylindrical cumulative coordination number $N(r)$ gives the number of molecules within a distance $r$ from the nanotube axis, and it is calculated using the integral ${ }^{26}$

$$
N(r)=\int_{0}^{r} \int_{0}^{2 \pi} \int_{-z_{1}}^{z_{2}} r^{\prime} d r^{\prime} d \varphi^{\prime} d z \rho\left(r^{\prime}, \varphi^{\prime}, z\right)
$$

with $r$ the radial distance from the nanotube axis, $-z_{1}$ and $z_{2}$ (with $z_{1,2}=1.5 \mathrm{~nm}$ for all the SWCNT in this work) the positions, with respect the axis center, of the two SWCNT extremes and $\rho\left(r^{\prime}, \varphi^{\prime}, z\right)$ the local number density of solute molecules. Note that only the molecules having the position of the CoM in the range $z_{1} \leq z \leq z_{2}$ are used in the calculation of the $\mathrm{N}(\mathrm{r})$ (as of the cRDF).

The cRDF is defined as

$$
c R D F(r)=\frac{N(r+\Delta r, h / 2)-N(r, h / 2)}{2 \pi r h \Delta r}=\frac{1}{\rho_{0}}\langle\rho(r, \varphi, z)\rangle_{\varphi, z}
$$

Where $\Delta r$ is a small increment of the radial distance, $\langle\ldots\rangle_{\varphi, z}$ is and average over $(0 \leq \varphi<2 \pi)$ and $(-1.5 \leq z \leq 1.5)$ at given $r$, and $\rho_{0}$ the density of the solvent in the bulk. Similarly with the spherical radial distribution function in our case $c R D F(r)$ is normalized in such a way that $c R D F(r) \rightarrow 1$ as $r \rightarrow \infty$. It gives the number of molecules of polymer units in the cylindrical volume within a distance $r$ from the SWCNT axis.

For DME and DMP the cRDF and $N(r)$ are calculated with respect the CoM of the molecules. For the polymers, they are calculated with respect the CoM of the ethylene (EO) and propylene (PO) oxide units composing the polymers with the first and last units including also the terminal group.

The density profile along the nanotube axial direction ${ }^{26}$ is defined as 
$\zeta(z)=\frac{1}{\pi \rho_{0} r^{2}} \int_{0}^{r} \int_{0}^{2 \pi} r^{\prime} d r^{\prime} d \varphi^{\prime} d z \rho\left(r^{\prime}, \varphi^{\prime}, z\right)$,
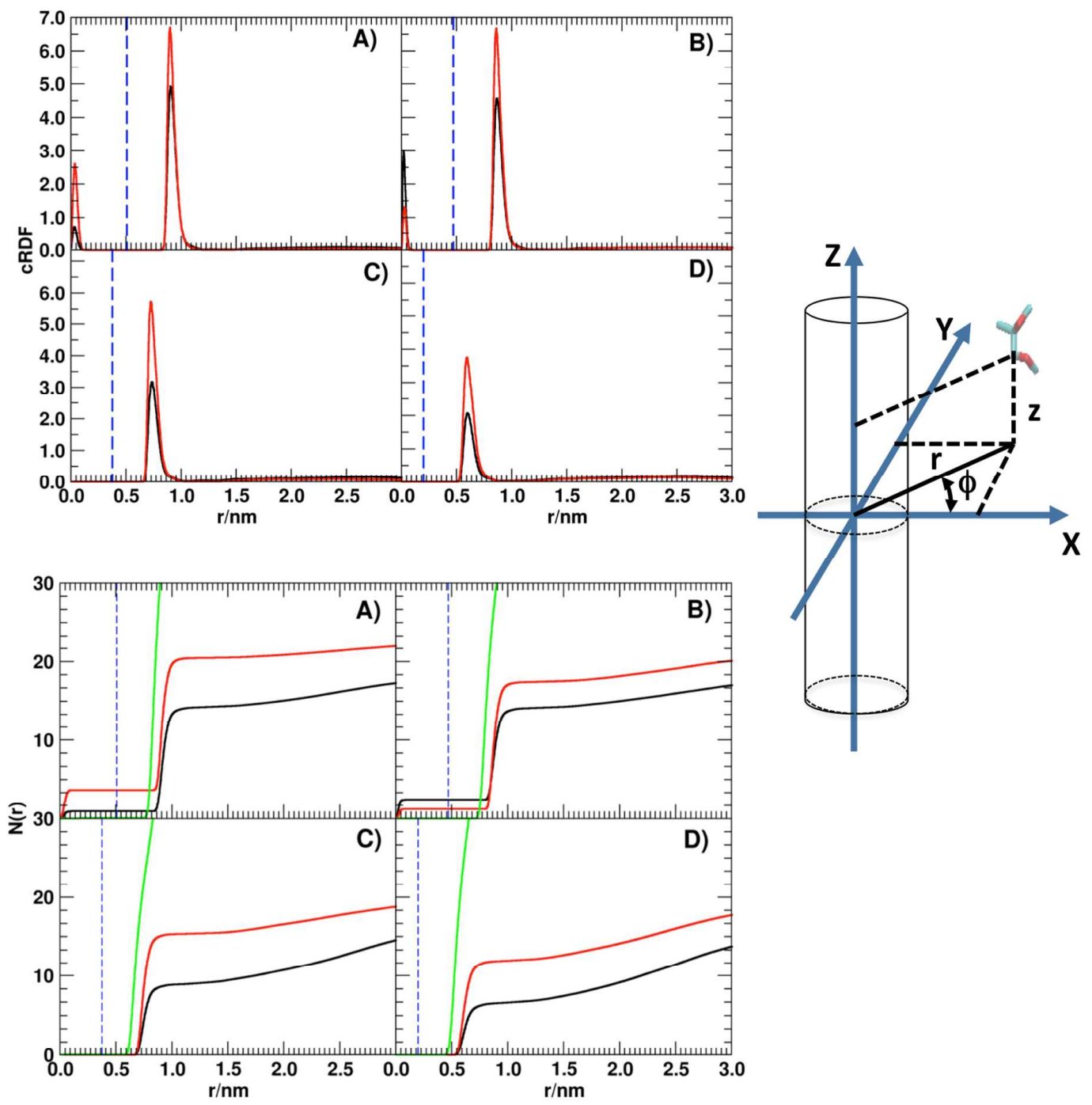

Figure 1. Top panel. cRDF for DME (black), DMP (red) molecules respect to the surface of SWCNT with chiral indices A) $(10,5), \mathrm{B})(7,7), \mathrm{C})(5,5)$ and D) $(5,0)$. The dashed vertical lines indicate the radius of the SWCNT. Bottom panel. Plots for the $N(r)$ of molecules of DME (black), DMP (red) and water (green) from the surface of the SWCNT with chiral indices A) $(10,5), B)(7,7)$, C) $(5,5)$ and D) $(5,0)$, respectively. The dashed vertical lines indicate the radius of the SWCNT. Right panel. Cylindrical coordinate system $(r, \varphi, z)$ used for the calculation of the cRDF. 
which calculate the density averaged along the cylindrical shell region between the nanotube axis $\left(r^{\prime}=0\right)$ and the nanotube radius $\left(r^{\prime}=r\right)$.

All simulations and the other analysis of the trajectories were performed using the GROMACS (version 4.5.5) software package (www.gromacs.org) ${ }^{27}$ and the visualization software VMD. ${ }^{28}$

\section{RESULTS AND DISCUSSION}

SWCNTS in DME and DMP Mixture. The coating effect of DME and DMP on the SWCNT surface was analyzed using the cRDF's of the solvent molecules with respect to the SWCNT surface. In Figure 1 (top panel), the cRDFs for the DME and DMP molecules are reported. The curves show two peaks. The small ones, located at $\sim 0.05 \mathrm{~nm}$ from the nanotube axis, are produced by molecules inside the nanotube. The other peaks, at $\sim 0.3 \mathrm{~nm}$, are generated by molecules coating the external surface of the SWCNT.

For all the simulations, cRDF peaks are higher for DMP than DME molecules, meaning a larger local density of the former one. The rapid decrease of the cRDF curves with the distance also indicates that the most of DME and DMP molecules bind the SWCNT.

In Figure 1 (bottom panel), the plots of $N(r)$ and, in Table 3, the number of DME and DMP molecules within $1.0 \mathrm{~nm}$ from the axis of each SWCNT are reported. It is evident from N(r) graphs that the number of DMP molecules at all distances from the surface of the $(10,5),(7,7)$ and $(5,5)$ SWCNT is always larger than DME ones. This tendency can be explained by the presence of a methyl group in the DMP that provides an additional interaction with the SWCNT compared to the DME. ${ }^{17}$ The number of DME and DMP at $1 \mathrm{~nm}$ distance of the nanotube axis is linearly proportional $\left(\mathrm{R}^{2}=0.96\right.$ and $\mathrm{R}^{2}=0.92$ from the liner regression fit for DMP and DME, respectively) to the surface are of the SWCNT (Table 3). The DMP/DME ratios at $N(r=1 n m)$ are larger for the smaller nanotubes than for the larger ones. The similar values of DMP/DME ratios for the two 
nanotube of similar diameter, $\operatorname{SWCNT}(7,7)$ and $\operatorname{SWCNT}(10,5)$, suggest that $\operatorname{SWCNT}$ chirality is not playing a important role in the surface distribution of the surfactants.

The hollow interior of both SWCNTs $(10,5)$ and $(7,7)$ was filled by a mixed file of DME and DMP molecules. A total number of 4.6 molecules were observed to fill in average the SWCNT $(10,5)$ cavity with a DMP/DME ratio of 3.9. In the SWCNT $(7,7)$, an average of 3.6 molecules was observed but in this case the DME molecules are more abundant than DMP (DMP/DME $=0.4$ in Table 3). In all four cases, no water molecules were observed inside the SWCNTs.

Table 3. Cylindrical cumulative coordination number $N(r)$ values for DME and DMP inside the SWCNT (In), and at the distance of $1.0 \mathrm{~nm}$ (Out) from the SWCNT axes. The DMP/DME radii indicated with the asterisk are calculated using the difference Out-In for comparison with the other two SWCNTs.

\begin{tabular}{|c|c|c|c|c|c|c|}
\hline \multirow{2}{*}{$\begin{array}{c}\text { SWCNT } \\
\text { chiral index }\end{array}$} & \multicolumn{2}{|c|}{$\mathbf{N}^{\mathbf{0}}$ DME } & \multicolumn{2}{c|}{$\mathbf{N}^{\mathbf{0}}$ DMP } & \multicolumn{2}{c|}{ DMP/DME } \\
\cline { 2 - 7 } & In & Out & In & Out & In & Out \\
\hline$(10,5)$ & $0.93 \pm 0.04$ & $12.4 \pm 0.2$ & $3.7 \pm 0.2$ & $19.7 \pm 0.2$ & 3.98 & $1.39^{*}$ \\
\hline$(7,7)$ & $2.45 \pm 0.07$ & $13.7 \pm 0.2$ & $1.21 \pm 0.05$ & $17.1 \pm 0.2$ & 0.49 & $1.41^{*}$ \\
\hline$(5,5)$ & 0 & $8.9 \pm 0.1$ & 0 & $15.3 \pm 0.2$ & $/$ & 1.71 \\
\hline$(5,0)$ & 0 & $6.6 \pm 0.1$ & 0 & $11.9 \pm 0.2$ & $/$ & 1.80 \\
\hline
\end{tabular}

In Figure 2, the normalized distributions of the molecules inside the SWCNT $(10,5)$ and $(7,7)$ are reported. Both DME and DMP molecules distribute with regular distribution patterns. For the SWCNT $(10,5)$, two DME peaks are observed inside the SWCNT and other four on both ends. DMP distribution shows five peaks inside the SWCNT and two small ones at the SWCNT ends. The positions of the peaks are similar to the DME ones. An additional peak at the SWCNT center $(\mathrm{z}=0)$ is observed for DMP but it is not present in DME. As from the values in Table 3, the density of DME molecules is lower than DMP. For the SWCNT $(7,7)$, the peaks for DME and DMP are in the same number (4 inside and 2 outside the SWCNT) and at the same locations, but higher for the DME than DMP, as expected from the average number of molecules observed in the SWCNT (see Table 3). In Figures $1 \mathrm{~S}$ and $2 \mathrm{~S}$ of SI, the time series diagram showing the occurrence times of single DME and DMP molecules inside the SWCNT $(10,5)$ and SWCNT $(7,7)$, respectively. 

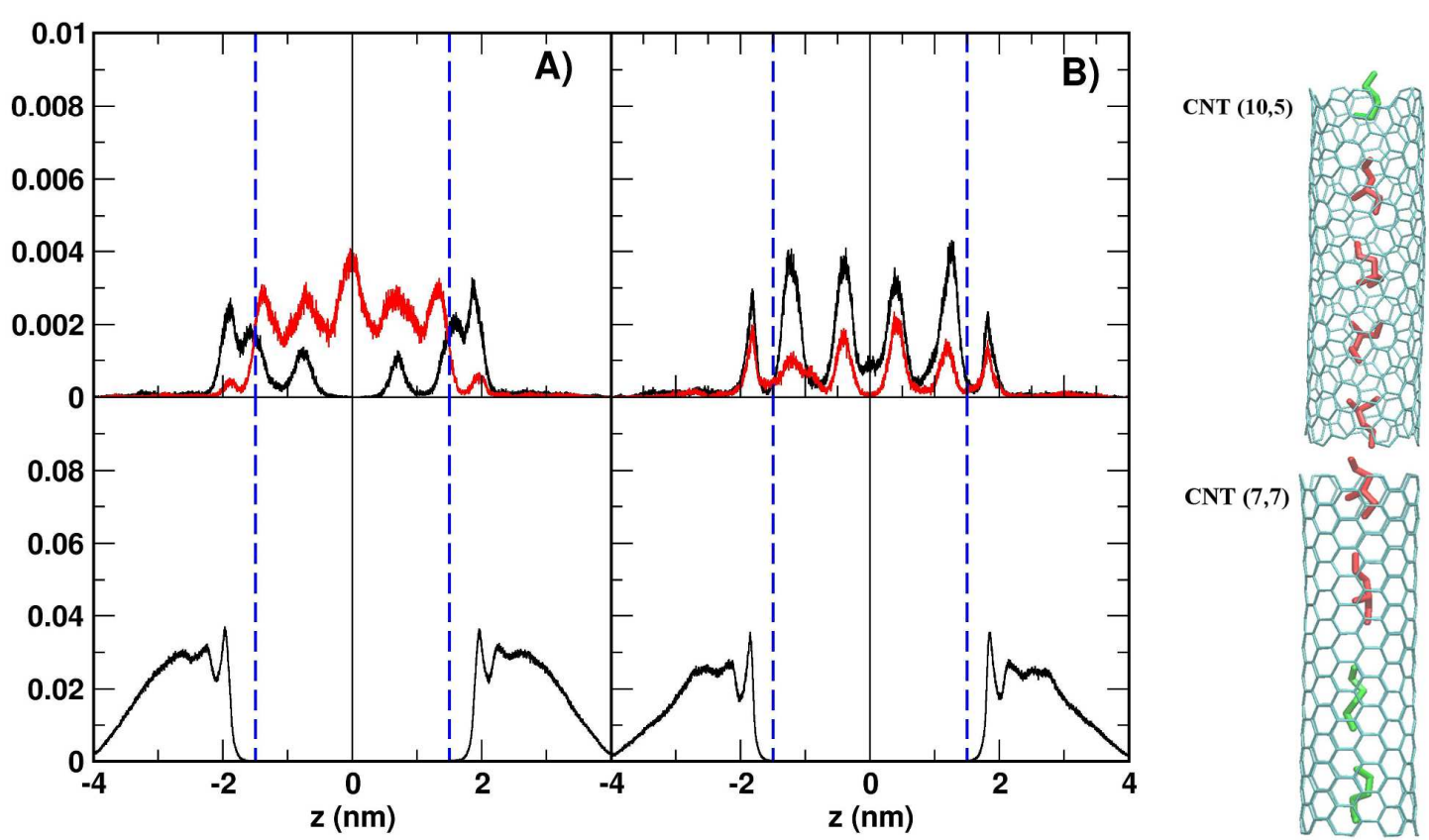

Figure 2. The density profile along the SWCNT axial direction, $\zeta(z)$, of DME, DMP (top graphs, black and red lines, respectively) and water (bottom graph) molecules distributed inside the SWCNT with chiral indices A) $(10,5)$ and B) $(7,7)$, respectively. The dashed lines indicate the SWCNT boundaries. At the right side are shown DME (green) and DMP (red) molecules inside the $\operatorname{SWCNT}(10,5)$ and $\operatorname{SWCNT}(7,7)$.

The residence time varies from just few nanoseconds to $25 \mathrm{~ns}$ (the whole simulation time). However, it is evident from the Figure $1 \mathrm{~S}$ and $2 \mathrm{~S}$ and from the low value of standard deviations reported in Table 3, that the total number of molecules that exchange with the bulk ones during the simulations is very small. For most of the simulation time, the molecules inside the SWCNT fluctuate around the peak positions of the $\zeta(z)$ distribution shown in Figure 2. Due to this long residence time, the DME/DMP distribution inside the nanotube is not preferential but randomly determined by the initial distribution of the molecules in the simulation box. For comparison the mean contact times of the DME/DMP molecules with the external surface of the nanotube are also reported in Table 1S of SI. The values range from 3-6 ns, and, as expected, slightly higher for the DMP than DME. 
Coating of the SWCNT (5,5) by PEO and PPO pentamers. In Figure 3 and Figure $3 \mathrm{~S}$ of the SI, the cRDF and $N(r)$ for EO and PO monomer units as a function of the distance from the central axes of the SWCNT $(5,5)$ are reported, respectively. The cRDFs show very similar position of the main peaks for both EO and PO units as observed for the DME/DMP ones. cRDF's of the EO units are characterized single peaked distributions. The the cRDF's of PO units for the isotactic isomer show a bimodal peck and an evident shoulder at $1 \mathrm{~nm}$ distance produced by the bulkier structure of the monomeric unit. In all the simulations, the $N(r)$ curves increase until a distance of $\sim 1 \mathrm{~nm}$ for then remain almost constant or grow very slowly. This behavior indicates that most of the polymers in solution are aggregated within $1 \mathrm{~nm}$ from the SWCNT axes. The value of $N(r)$ at $\mathrm{r}=1 \mathrm{~nm}$, for the $\mathrm{EO}$ units in the $\mathrm{S}(5,5) \mathrm{EO}_{5}$ simulation is $46.5 \pm 0.6$ ( $\sim 9.3$ PEO chains). For the two $\mathrm{S}(5,5) \mathrm{PPO}_{5}$ simulations, the values of $\mathrm{N}(\mathrm{r})$ are larger than the $\mathrm{PEO}_{5}$ one and equal to $50.1 \pm 0.5$ and $50.8 \pm 0.5$ (corresponding to $10 \mathrm{PPO}$ chains for both of them) for the isotactic (RRRRR) and syndiotactic $(R S R S R)$ polymers, respectively.

For the PEO/PPO mixtures, an interesting effect on the preferential binding determined by the $\mathrm{PPO}_{5}$ chain conformation has been observed. In Table 4 , the values of $N(r=1 n m)$ from all the polymer mixture simulations are reported. For the mixture simulation of the three isomers $\mathrm{PPO}_{5}(R R R R R)$, $\mathrm{PPO}_{5}($ RRSRR $)$ and $\mathrm{PPO}_{5}(R S S R S)$, the sum of the $\mathrm{PEO}_{5}$ and $\mathrm{PPO}_{5}$ chains on the SWCNT surface is equal to the one from the single polymer simulations (9-10), but the $\mathrm{PEO}_{5}$ chains are in larger amount with respect to the $\mathrm{PPO}_{5}$ ones. On the contrary, for the mixture with the isomers $\mathrm{PPO}_{5}(R S R S R)$ and $\mathrm{PPO}_{5}($ RRSRS), the order of preferential binding is again in agreement with the one observed for the DME/DMP mixtures. 

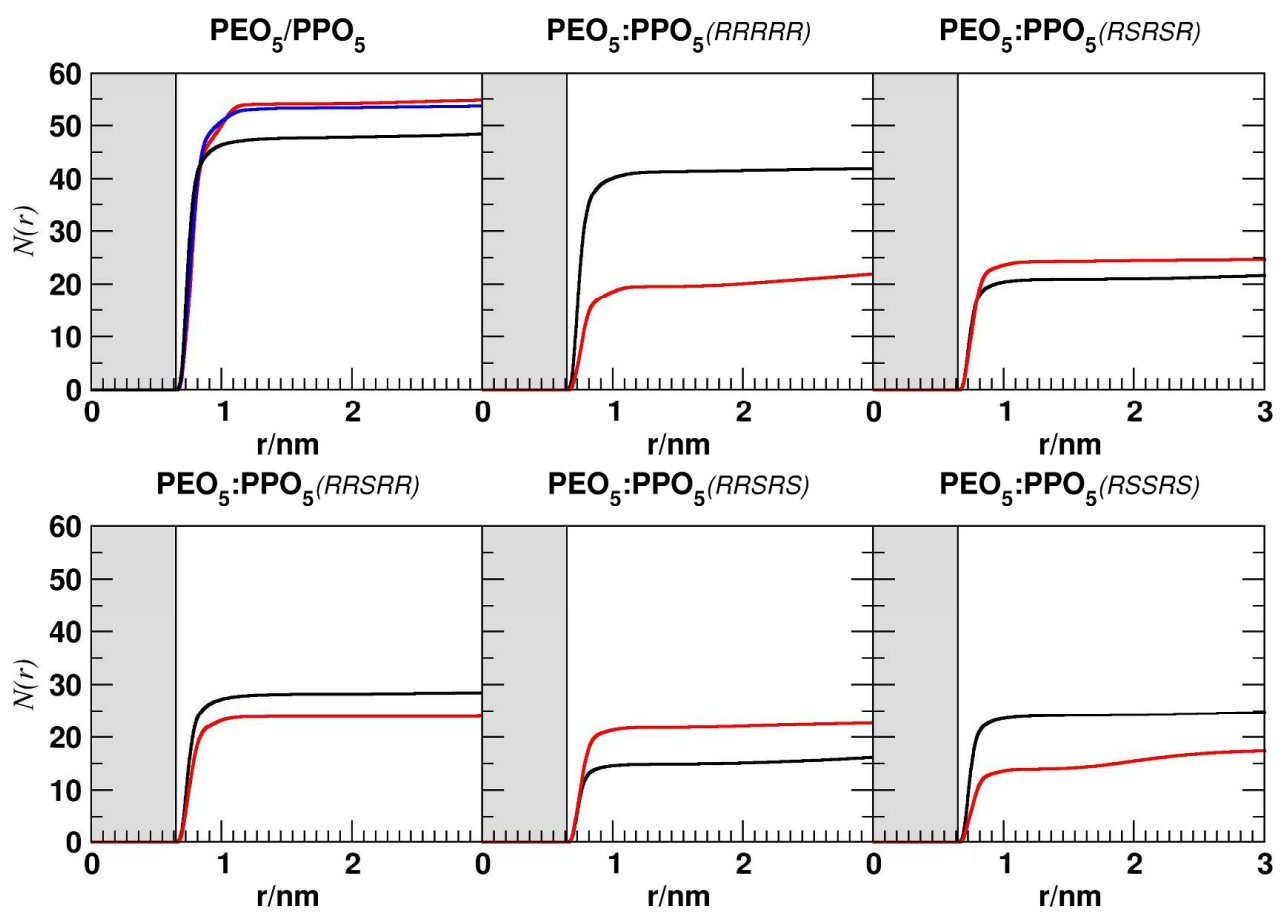

Figure 3. Plots of the $N(r)$ of PO monomers as function of the distance from the SWCNT $(5,5)$ (grey bands) axis for the water solutions of $\mathrm{PEO}_{5}$ (black), and $\mathrm{PPO}_{5}$ isotactic (red) and syndiotactic (blue). The plot of $\mathrm{N}(\mathrm{r})$ for the $50 \%$ mixture of the two polymers with different $\mathrm{PPO}_{5}$ isomers are reported in the other panels with EO and PO represented using back and red lines, respectively.

The possible culprit of the observed differences in binding behavior of the $\mathrm{PPO}_{5}$ isomers could be an entropic effect determined by distinct conformations assumed by polymer chains in solution. To verify this hypothesis, the radius of gyration $(\mathrm{Rg})$ and the end-to-end distance of the polymers have been calculated and reported in Table 4. For the $\mathrm{PEO}_{5}$, an average value of $0.42 \pm 0.01 \mathrm{~nm}$ is observed in all the simulations. These values are consistent with those observed in MD simulations of the same $\mathrm{PEO}_{5}$ and $\mathrm{PPO}_{5}$ chains in absence of the SWCNT indicating that the presence of the SWCNT do not affect the average conformation of the polymer in solution that, henceforth, is primarily determined by its stereochemistry. ${ }^{18}$ 
Table 4. Cylindrical cumulative coordination number $N(r)$ of the EO and PO units calculated at $1 \mathrm{~nm}$ of distance from the SWCNT(5,5) axis from the simulations of the $\mathrm{PEO}_{5}: \mathrm{PPO}_{5}$ mixture of the different $\mathrm{PPO}_{5}$ isomers. N.C. stands for number of chains and it is calculated by dividing the $\mathrm{N}(\mathrm{r}-1 \mathrm{~nm})$ by 5 . The average radius of gyration $(<\mathrm{Rg}>)$ and end to end distance $(<\mathrm{EtoE}>)$ for the PPO chains are reported in the last two columns, respectively.

\begin{tabular}{|c|c|c|c|c|c|c|c|}
\hline Simulation & $\begin{array}{l}\text { EO units } \\
N(r=1 \mathrm{~nm})\end{array}$ & N.C. & $\begin{array}{l}\text { PO units } \\
N(r=1 n m)\end{array}$ & N.C. & C.S. & $\begin{array}{c}\mathbf{P P O}_{5} \\
<\mathbf{R g}>/ \mathbf{n m}\end{array}$ & $\begin{array}{c}\text { PPO }_{5} \\
<\text { EtoE }>/ \text { nm }\end{array}$ \\
\hline $\mathrm{S}(5,5) \mathrm{EO}_{5}: \mathrm{PO}_{5}(R R R R R)$ & $29.1 \pm 0.4$ & 5.8 & $15.2 \pm 0.3$ & 3.0 & 8.8 & $0.36 \pm 0.01$ & $1.00 \pm 0.05$ \\
\hline $\mathrm{S}(5,5) \mathrm{EO}_{5}: \mathrm{PO}_{5}(R S R S R)$ & $20.4 \pm 0.4$ & 4.1 & $23.6 \pm 0.3$ & 4.7 & 8.8 & $0.41 \pm 0.01$ & $1.19 \pm 0.08$ \\
\hline$\overline{\mathrm{S}(5,5) \mathrm{EO}_{5}: \mathrm{PO}_{5}(\mathrm{RRSRR})}$ & $27.2 \pm 0.4$ & 5.4 & $23.2 \pm 0.3$ & 4.6 & 10 & $0.40 \pm 0.01$ & $1.06 \pm 0.08$ \\
\hline$\overline{\mathrm{S}(5,5) \mathrm{EO}_{5}: \mathrm{PO}_{5}(\mathrm{RRSRS})}$ & $14.6 \pm 0.3$ & 2.9 & $21.4 \pm 0.3$ & 4.3 & 7.2 & $0.41 \pm 0.01$ & $1.13 \pm 0.08$ \\
\hline $\mathrm{S}(5,5) \mathrm{EO}_{5}: \mathrm{PO}_{5}(R S S R S)$ & $23.6 \pm 0.3$ & 4.7 & $13.6 \pm 0.3$ & 2.7 & 7.4 & $0.39 \pm 0.01$ & $1.05 \pm 0.09$ \\
\hline
\end{tabular}

In Figure 4S of SI, the $\mathrm{Rg}$ distributions for the $\mathrm{PEO}_{5}$ and the different $\mathrm{PPO}_{5}$ isomers from the mixture simulations are also shown. The isomers $\mathrm{PPO}_{5}(R R R R R), \mathrm{PPO}_{5}(R R S R R)$ and $\mathrm{PPO}_{5}(R S S R S)$ with a weaker SWCNT binding capability than $\mathrm{PEO}_{5}$, have very similar $\mathrm{Rg}$ values $(\sim 0.4 \mathrm{~nm})$. On the contrary, the other two isomers with higher binding capability than $\mathrm{PEO}_{5}$, have a more extended conformation (average $\mathrm{Rg}$ close to the one of $\mathrm{PEO}_{5}$ chains). As representative examples, in Figure S4, the structures of the polymer bound to the SWCNT at the end of the of the $\mathrm{S}(5,5) \mathrm{EO}_{5}: \mathrm{PO}_{5}(R R R R R)$ and $\mathrm{S}(5,5) \mathrm{EO}_{5}: \mathrm{PO}_{5}(R S R S R)$ simulations are shown. The isotactic $\mathrm{PPO}$ pentamers (on the left) are more compact and distributed in the middle of the SWCNT compared to the syndiotactic ones (on the right).

In summary, the results of the simulations have shown that the more compact structures of the isotactic and the other two atactic polymers reduces their capability to bind the nanotube surface 
with respect to the $\mathrm{PEO}_{5}$. A possible explanation of this behavior is that the more compact polymeric structures are penalized in the number of atomic interaction with the SWCNT surface compared to the more extended PEO chains. The later being more flexible can adapt better to the SWCNT surface despite their more hydrophilic nature.

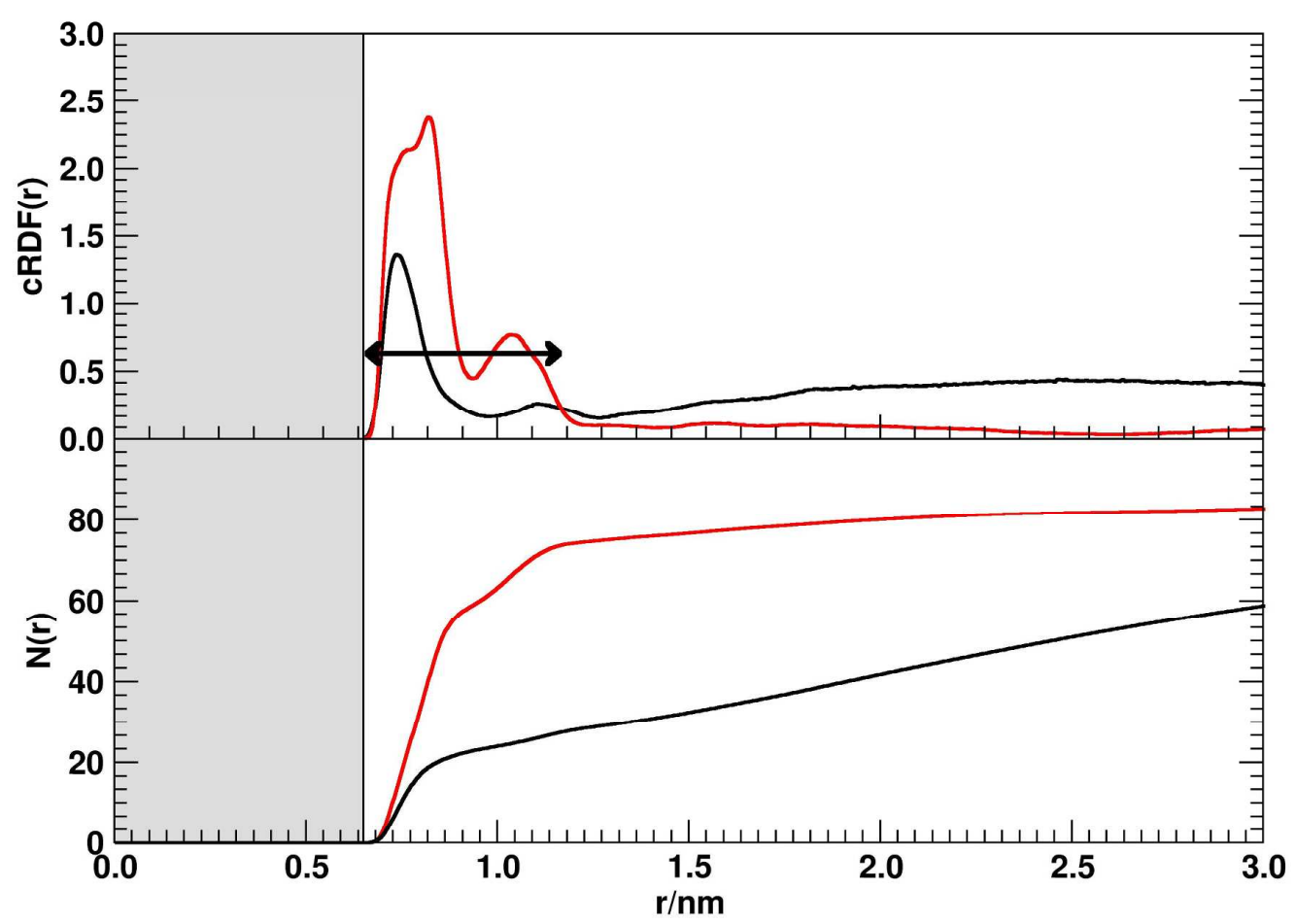

Figure 4. Plots of the cRDF (top) and $N(r)$ of EO (black curve) and PO (red lines) monomers (bottom) of the polaxamer L64 versus the SWCNT $(5,5)$ (grey bands) axis. The double arrow in the cRDF plot indicates the extension of the shell (PO monomers) region around the SWCNT.

Interaction of L64 triblock copolymer with SWCNT. The average radius of gyration of the polymers is $1.79 \pm 0.15 \mathrm{~nm}$, respectively. These value is slightly smaller than the corresponding values of $1.86 \pm 0.2 \mathrm{~nm}$ obtained from a simulation of the polymer in the same water box but without the SWCNT. The latter agree very well with the experimental SANS data of $1.8 \mathrm{~nm}$ determined for L64 unimers at $281 \mathrm{~K}$ in $\mathrm{D}_{2} \mathrm{O}^{29}$ 
In Figure 4, the cRDF and $N(r)$ for PEO and PPO units of the L64 polymer as a function of the distance from central axes of SWCNT $(5,5)$ are reported. The cRDFs show the larger tendency of PPO than PEO units to bind the SWCNT surface. The cRDF of PPO units is multimodal and it is characterized by a large peak at $\sim 0.75 \mathrm{~nm}$, together with a broad shoulder at shorter distance, and a smaller peak at $1.0 \mathrm{~nm}$. The cRDF of EO monomers shows also the presence of two single-modal peaks located at the same positions as the PO ones. The PO's $N(r)$ curve shows a rapid increase within $0.65 \mathrm{~nm}$ from the SWCNT surface, followed by a plateau. On the contrary the curve corresponding to EO monomers after an initial fast growth, it continue to increase almost linearly. The $N(r)$ within the distance of $1.35 \mathrm{~nm}$ from the central axes of SWCNT for the EO and PO units is equal to $30.1 \pm 0.4,75.6 \pm 0.6$, respectively.

In Figure 5, snapshots at the beginning and after $75 \mathrm{~ns}$ from the simulation of L64 triblock copolymer in the presence of a SWCNT $(5,5)$ are reported. In the initial configuration (Figure 5A), the six L64 chains (obtained from previous simulation in water ${ }^{18}$ ) are regularly arranged around the SWCNT. During the simulation four out of six L64 chains are in contact mainly with the more hydrophobic PPO blocks to the SWCNT surface, and the more hydrophilic PEO blocks extending in the bulk water (Figure 5, B and C). From the $N(r)$ at $1 \mathrm{~nm}$ from the surface of the SWCNT (1.35 $\mathrm{nm}$ from the axis), it is clear that $2.5(=76 / 30)$ of these 4 chains are in direct contact with the lateral surface of the nanotube and form a core extending for $\sim 1 \mathrm{~nm}$ from the SWCNT surface. 


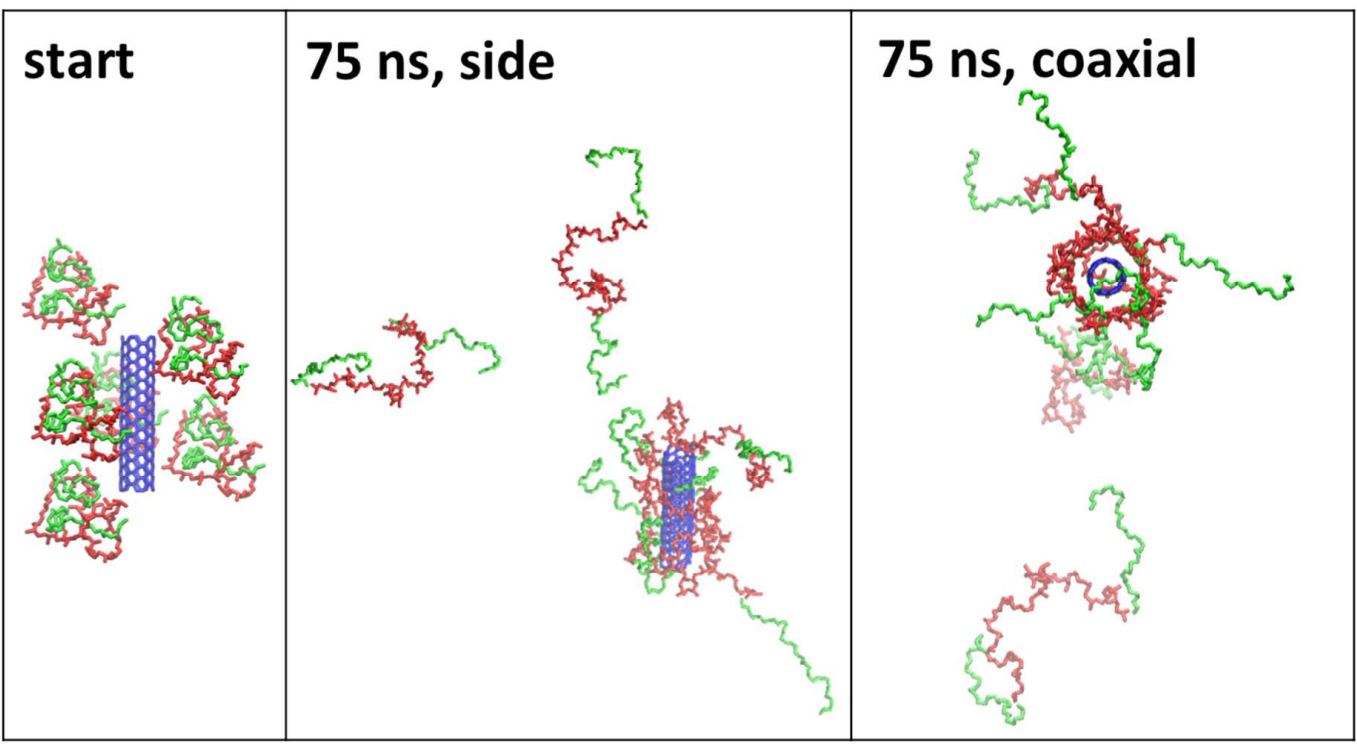

Figure 5. SWCNT $(5,5)$ with six L64 triblock copolymer chains. The starting configuration and two different views of the same configuration at $75 \mathrm{~ns}$ are shown. The hydrophilic (PEO) and hydrophobic (PPO) blocks of L64 are shown in green and in red, respectively. For clarity, water molecules are omitted.

The results of our simulations provide a further support to the so-called non-wrapping mechanism of adsorption of block copolymers to SWCNT's and MWCNT's. ${ }^{12}$ This study has evidenced the tendency of the PPO block to absorb preferential of PEO blocks. More recent small angle scattering studies of the linear polymers and surfactants including poloxamers have also shown that the ratio of PEO and PPO determine the capability of the surfactant to solubilize the SWCNT. ${ }^{30}$ SANS studies of larger Pluronics F127 and F108 have also proposed a core-shell-chain model of polymerSWCNT interaction based on the experimental data. ${ }^{13,31}$ The results of our simulations with the Pluronics L64 qualitatively agree with this model. In particular, the cRDF analysis (see Figure 4) indicates that polymer chains bind the nanotube and coat the surface mostly with the PPO units. The PPO units observed at the end of our simulation (Figure 6S of the SI) are partially wrapped around the nanotube forming a shell layer tightly bound to the SWCNT. The PEO blocks protrude out of the surface forming a more hydrophilic corona around the shell that extend into the aqueous media. 
This is consistent with the core-shell-chain model proposed by Granite et al for longer polaxamers. ${ }^{13,31}$

Effect of Polaxamer L64 on the SWCNT Bundle Formation. To the best of our knowledge, direct structural experimental measurements (e.g. SANS) for the polaxamer L64 are not yet available. However, experimental data on the capability of polaxamers to disperse SWCNT bundles are available in literature. ${ }^{12-13,}$ 30-33 Therefore, we have performed a simulation of 3 isolated SWCNT $(5,5)$ with and without polymers to assess the effect of the polaxamer L64 on the formation of a SWCNT bundle. A bundle consisting of three SWCNT of $3 \mathrm{~nm}$ long has been obtained placing nanotubes into simulation box, filled with water molecules (see Figure 7S A and B in SI) and running $100 \mathrm{~ns}$ simulation. After $80 \mathrm{~ns}$, the three SWCNTs formed a bundle having all SWCNTs perfectly aligned and packed with an average minimum distance of $\sim 0.36 \mathrm{~nm}$. 
1 2 3 4 5 6 7 8 9 10 11 12 13 14 15 16 17 18
A

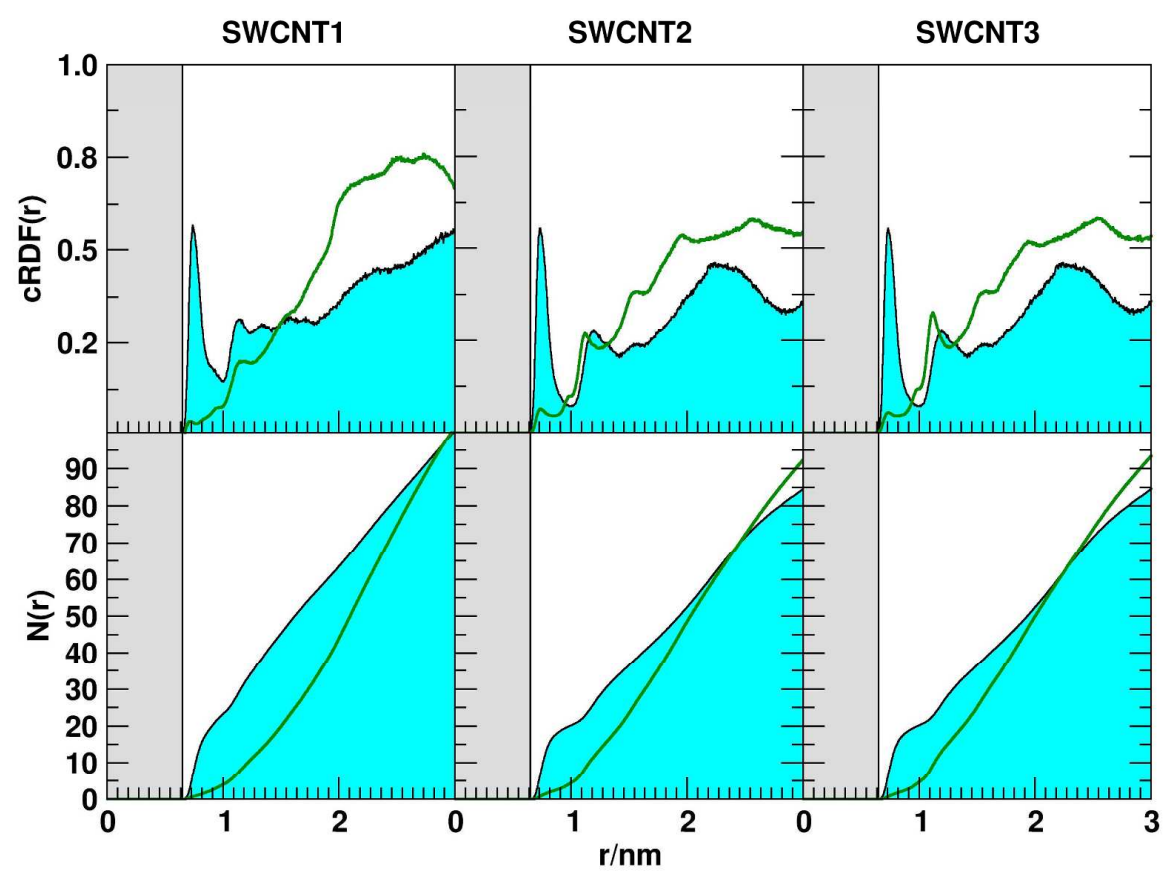

B

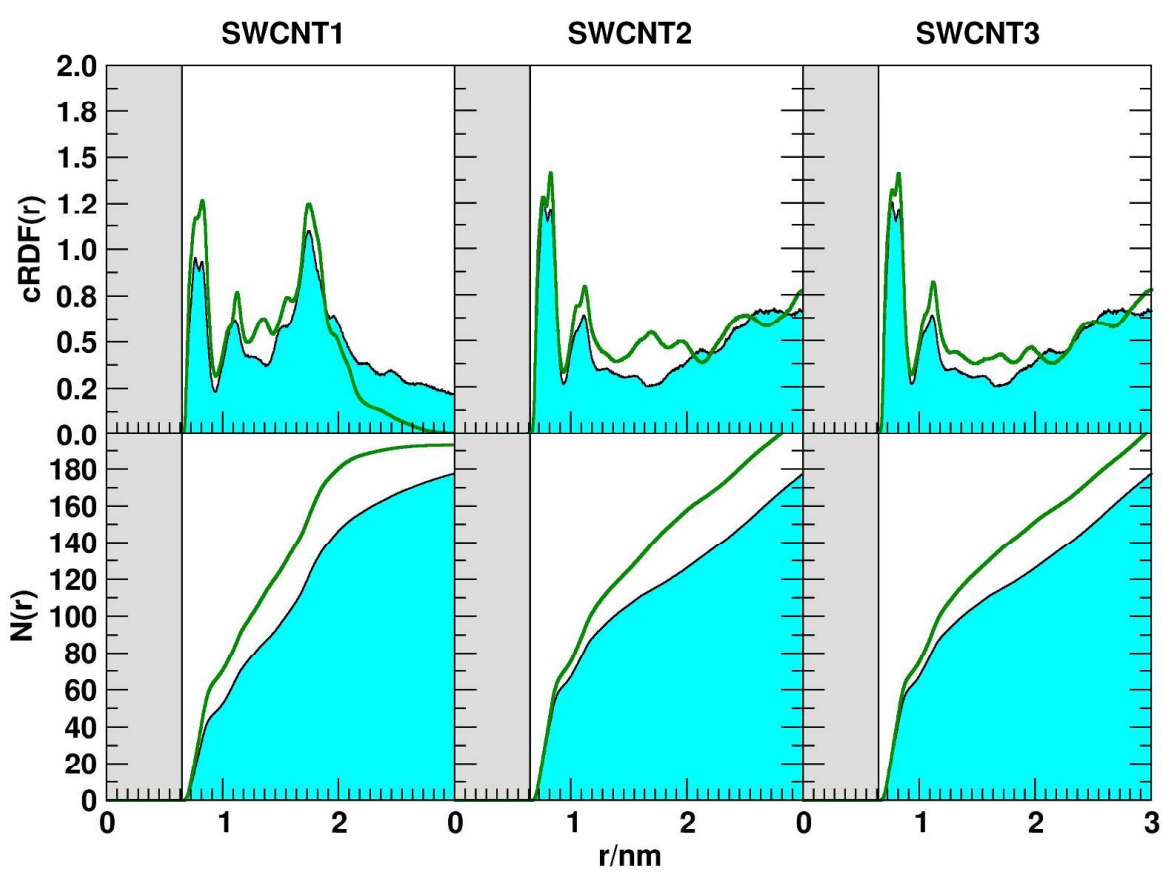

Figure 6. Plots of the cRDF's (top panels) and $N(r)$ 's (lower panels) of PEO (A) and PPO (B) monomers of the polaxamer L64 around the three SWCNT $(5,5)$. The filled cyan curves are calculated in the time frame 0$10 \mathrm{~ns}$, the dark green line in the last $50 \mathrm{~ns}$ of the simulations. The grey bands indicate the SWCNT radius. 
The effect of the L64 triblock copolymers on the spontaneous aggregation of the SWCNTs was studied by placing into a simulation box, three replica of surface coated SWCNT obtained from the simulation of the isolated SWCNT with six L64 polymers. In the Figure 7S of the SI the starting configuration of the simulation with three SWCNTs having the surface covered by L64 copolymers into a water box is shown. After 10 ns of simulation, two out of three SWCNT start to aggregate and they remain close and aligned for the rest of the simulation (see Figure 8S).

In Figure 6, for each SWCNT, the $c R D F$ 's (top panels) and $N(r)$ 's (bottom panels) for EO (Figure 6A) and PO (Figure 6B) units of the L64 polymers as a function of the distance from central axes of SWCNT $(5,5)$ are reported. The filled curves in cyan colour are calculated in the time frame 0-10 ns, the dark green line in the last 50 ns of the simulation. For all the SWCNT, the first peak in the cRDFs (Figure 6A, top panels) is considerably reduced in the last $50 \mathrm{~ns}$ of the simulation compared to the beginning as the units are depleted from the SWCNT surface (Figure 6B, bottom panel). On the contrary, the PO monomers accumulate more around the nanotube than the PEO ones, as shown by the increase of the peaks in the cRDF (Figure 6B, top panels) and by the $N(r)$ curves (Figure 6B, bottom panels). In this case, only the $N(r)$ curve for the SWCNT1 reaches a plateau in the last $50 \mathrm{~ns}$. In Figure 7, the process of SWCNT aggregation is described by the fraction of their atomic contacts relative to the average number of and uncoated SWCNT bundle. SWCNT1 and SWCNT2 aggregate at the beginning of simulation, the number of contact is slightly less then in the uncoated CNT bundle for the presence of the polymers (see also Figure 8S) although the average minimum distance $(\sim 0.36 \mathrm{~nm})$ between these two nanotubes corresponds to the average value observed in the uncoated nanotube in pure water. The SWCNT3 starts to form a partial contact ( 20\%) with SWCNT1 at $\sim 10$ ns that remain until the end of the simulation. The same nanotube forms a much less number of relative contacts $(\sim 10 \%)$ with the SWCNT2 but mainly in the interval $57-72 \mathrm{~ns}$ (see also Figure 5S). 
This result is in qualitative agreement with the results presented in previous experimental work. ${ }^{32,34}$ In particular, Shvartzman-Cohen have used differential scanning calorimetry and spin-probe electron paramagnetic resonance to probe the dynamics of the different Pluronics in the presence of $\mathrm{CNTs}^{23}$. More recently, Xin et al. ${ }^{32}$ they have studied the dispersion of the nanotube by different amphiphilic block copolymers, such as F127, the star-like block copolymer AP432 and L64, using by UV-VIS-NIR measurements and Raman spectroscopy techniques. ${ }^{32}$ In their work, it was observed that the longer Pluronics F127 has good capabilities to disperse CNTs, while the L64 was unable to produce a good dispersion in water. They have explained this phenomenon with the shorter hydrophilic (PEO) chains of L64 in comparison to the F127 (and AP432 one), which accounted for the weak steric repulsion between the individual nanotubes in the CNT bundles. Our results show that the L64 chains are easily displaced from the nanotube surface by the other nanotubes.

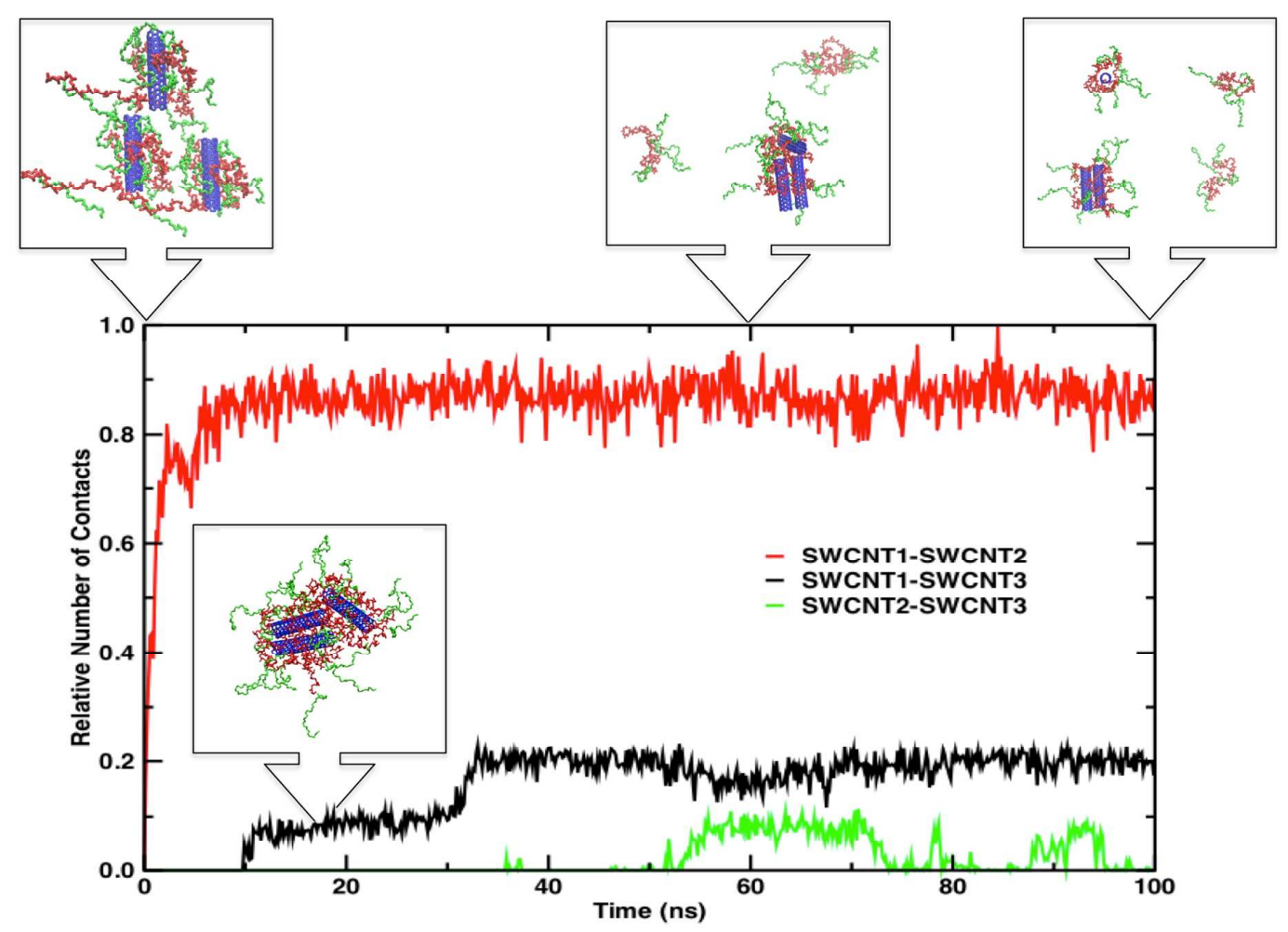

Figure 7. On the left panel, minimum distances between the SWCNT1, SWCNT2 and SWCNT3. On the right panel, fraction of the number of contacts within $0.6 \mathrm{~nm}$ between the SWCNT's and relative to the average number of and uncoated SWCNT bundle. 


\section{CONCLUSIONS}

Atomistic MD simulations have been performed to study the interaction of the linear ether based polymers with SWCNT. Based on the results of these simulations, we have shown the tendency of the DMP molecules to preferentially solvate the SWCNT surface than DME molecules. This effect seems not influenced by the chirality of nanotube, but only by its diameter. We have also observed in our simulations, the diffusion of both DMP and DME inside the two larger nanotubes SWCNT $(10,5)$ and $\operatorname{SWCNT}(7,7)$. In our simulations, the molecules inside the SWCNT have a low exchange with the bulk molecules (preferentially absorbed on the SWCNT surfaces), and, therefore, their distribution resulted not preferential but randomly determined by the initial distribution of the molecules in the simulation box. An interesting differential binding to the $\operatorname{SWCNT}(5,5)$ surface was observed in the mixtures of PEO and PPO pentamers. The order of preferential binding of the two polymers to the SWCNT is strongly dependent of the stereoisomeric structure of the $\mathrm{PPO}_{5}$ chain. We have observed that this entropic effect can be correlated to the compactness of the polymer measured by the radius of gyration. PPO isomers with radius of gyration close to the $\mathrm{PEO}_{5}$ chains retain the order of preferential binding of the SWCNT as observed in the DME/DMP oligomers. More compact chains have an inverted order of SWCNT binding capability. These interesting results need to be further investigated to better understand the nature of this effect and the dependence by other parameters as for example the nanotube dimensionality ${ }^{15}$ and/or the polymer size. Unfortunately, to the best of our knowledge, no experimental data are available in the literature concerning interaction of the short PEO/PPO oligomers with SWCNT, and we hope that these results can also stimulate further experimental studies. The results of the simulations with triblock copolymer L64 has shown that the polymer chains bind the nanotube surface with PPO block regions, while the PEO block of the polymers extend in the bulk water. This result provide an atomistic model of CNT coating in agreement with non-wrapping interaction mechanism ${ }^{12}$ and the core-shell-chains model proposed by Granite et al. for polaxamers on the base of experimental measurements. 
Finally, it was shown that the coating by L64 of the SWCNT surface of SWCNT is not stable in presence of other SWCNT. The detachments of the chains from isolated coated nanotubes resulted in the aggregation of two of them. This last result is in qualitative agreement with experimental evidences of the weaker capability of the Pluronic L64 to disperse CNT bundles. Nevertheless, the short size of the SWCNT, the concentration of polymers chains, and the time scale of the simulation limit the results of model of the coating process. From a thermodynamics perspective, there exist thermodynamics theories that can partly address some of the aforementioned limitations as those related to the concentration of polymers required to stabilize a dispersion of SWCNT (see ref. 12 and 15). For the molecular details, more extensive simulation studies at different level of scales are needed to give a more extensive description of the coating process for polymers and SWCNT of different length and concentration.

In summary, the computational study reported in this paper gives a detailed account at molecular level of the coating mechanism of polyether-based polymers and block copolymers to SWCNT. The results confirm previously proposed coating mechanisms, and reveal new molecular aspects on the effect of polymer conformation on their absorption to SWCNT surface that needs further experimental and theoretical investigations. In particular, coarse-grained models ${ }^{35-38}$ developed in our groups will allow the study of these processes on larger length of scales giving a more quantitative assessment of the coating mechanisms with longer SWCNT and polymers. The understanding of this process is important for the applications in medicine and biotechnology. For example, for the design novel CNT based drug carriers and/or for assess the biological toxicity of CNT.

Supporting Information Available: Scheme with the chemical formulas for the polymers used in this study. Distribution of DME and DMP molecules inside the CNT(10,5) and CNT(7,7). Cylindrical RDF distributions of both PEO and PPO pentamers. Distributions of the radii of gyration for the $\mathrm{PEO}_{5}$ and different isomers of $\mathrm{PPO}_{5}$. Conformations of $\mathrm{PPO}_{5}$ and $\mathrm{PPO}_{5}$ polymer 
chains bound to the SWCNT $(5,5)$ at the end of the simulations. Conformations of L64 polymer chains bound to the SWCNT $(5,5)$ at the end of the simulations. SWCNT $(5,5)$ bundle formation in water. Snapshots from the $3 \mathrm{~S}(5,5) \mathrm{L} 64$ simulation. This material is available free of charge via the Internet at http://pubs.acs.org.

\section{ACKNOWLEDGMENTS}

This work was performed using the computer facilities of the Computational Laboratory for Analysis, Modeling and Visualization (CLAMV) at Jacobs University Bremen. E.S. thanks the international Ph.D. program in nanoscience and nanotechnology between the University of Salerno and Jacobs University Bremen for the financial support.

\section{REFERENCES}

1. Porter, A. E.; Gass, M.; Muller, K.; Skepper, J. N.; Midgley, P. A.; Welland, M., Direct imaging of single-walled carbon nanotubes in cells. Nat. Nanotechnol. 2007, 2 (11), 713-717.

2. Prato, M.; Kostarelos, K.; Bianco, A., Functionalized carbon nanotubes in drug design and discovery. Acc. Chem. Res. 2007, 41 (1), 60-68.

3. Bhirde, A. A.; Patel, V.; Gavard, J.; Zhang, G.; Sousa, A. A.; Masedunskas, A.; Leapman, R. D.; Weigert, R.; Gutkind, J. S.; Rusling, J. F., Targeted killing of cancer cells in vivo and in vitro with EGF-directed carbon nanotube-based drug delivery. Acs Nano 2009, 3 (2), 307316.

4. Thess, A.; Lee, R.; Nikolaev, P.; Dai, H.; Petit, P.; Robert, J.; Xu, C.; Lee, Y. H.; Kim, S. G.; Rinzler, A. G., Crystalline ropes of metallic carbon nanotubes. Science 1996, 273 (5274), 483-487.

5. Komatsu, N.; Wang, F., A Comprehensive Review on Separation Methods and Techniques for Single-Walled Carbon Nanotubes. Materials 2010, 3 (7), 3818-3844.

6. Hersam, M. C., Progress towards monodisperse single-walled carbon nanotubes. Nat. Nanotechnol. 2008, 3 (7), 387-394.

7. Liu, Z.; Tabakman, S.; Welsher, K.; Dai, H., Carbon nanotubes in biology and medicine: in vitro and in vivo detection, imaging and drug delivery. Nano research 2009, 2 (2), 85-120.

8. Kostarelos, K.; Bianco, A.; Prato, M., Promises, facts and challenges for carbon nanotubes in imaging and therapeutics. Nat. Nanotechnol. 2009, 4 (10), 627-633.

9. Gong, H.; Xu, G.; Liu, T.; Pang, J.; Dou, W.; Xin, X., Synthesis of block polyethers with various structures and their application in dispersing single-walled carbon nanotubes. Colloid. Polym. Sci. 2011, 289 (8), 933-942.

10. Blanch, A. J.; Lenehan, C. E.; Quinton, J. S., Optimizing surfactant concentrations for dispersion of single-walled carbon nanotubes in aqueous solution. J. Phys. Chem. B 2010, 114 (30), 9805-9811. 
11. Nagarajan, R.; Bradley, R. A.; Nair, B. R., Thermodynamically stable, size selective solubilization of carbon nanotubes in aqueous solutions of amphiphilic block copolymers. $J$. Chem. Phys. 2009, 131, 104906.

12. Nativ-Roth, E.; Shvartzman-Cohen, R.; Bounioux, C. 1.; Florent, M.; Zhang, D.; Szleifer, I.; Yerushalmi-Rozen, R., Physical Adsorption of Block Copolymers to SWNT and MWNT: A Nonwrapping Mechanism. Macromolecules 2007, 40 (10), 3676-3685.

13. Granite, M.; Radulescu, A.; Pyckhout-Hintzen, W.; Cohen, Y., Interactions between Block Copolymers and Single-Walled Carbon Nanotubes in Aqueous Solutions: A Small-Angle Neutron Scattering Study. Langmuir 2011, 27 (2), 751-759.

14. Frise, A. E.; Pagès, G.; Shtein, M.; Pri Bar, I.; Regev, O.; Furó, I. n., Polymer Binding to Carbon Nanotubes in Aqueous Dispersions: Residence Time on the Nanotube Surface As Obtained by NMR Diffusometry. J. Phys. Chem. B 2012, 116 (9), 2635-2642.

15. Szleifer, I.; Yerushalmi-Rozen, R., Polymers and carbon nanotubes - dimensionality, interactions and nanotechnology. Polymer 2005, 46 (19), 7803-7818.

16. Yang, M.; Koutsos, V.; Zaiser, M., Interactions between polymers and carbon nanotubes: a molecular dynamics study. J. Physical Chem. B 2005, 109 (20), 10009-10014.

17. Hezaveh, S.; Samanta, S.; Milano, G.; Roccatano, D., Structure and Dynamics of 1,2dimethoxyethane and 1, 2-dimethoxypropane in Aqueous and Non-aqueous Solutions: A Molecular Dynamics Study. J. Chem. Phys. 2011, 135 (16), 164501-164511.

18. Hezaveh, S.; Samanta, S.; Milano, G.; Roccatano, D., Molecular dynamics simulation study of solvent effects on conformation and dynamics of polyethylene oxide and polypropylene oxide chains in water and in common organic solvents. J. Chem. Phys. 2012, 136 (12), 124901-12.

19. Walther, J. H.; Jaffe, R.; Halicioglu, T.; Koumoutsakos, P., Carbon nanotubes in water: structural characteristics and energetics. J. Phys. Chem. B 2001, 105 (41), 9980-9987.

20. Werder, T.; Walther, J. H.; Jaffe, R.; Halicioglu, T.; Koumoutsakos, P., On the water-carbon interaction for use in molecular dynamics simulations of graphite and carbon nanotubes. $J$. Phys. Chem. B 2003, 107 (6), 1345-1352.

21. van der Spoel, D.; Lindahl, E.; Hess, B.; Kutzner, C.; van Buuren, A. R.; Apol, E.; Meulenhoff, P. J.; Tieleman, D. P.; Sijbers, A. L. T. M.; Feenstra, K. A.; van Drunen, R.; Berendsen, H. J. C., Gromacs User Manual version 4.0. 2005.

22. Berendsen, H. J. C.; Grigera, J. R.; Straatsma, T. P., The Missing Term in Effective Pair Potentials. J. Phys. Chem. 1987, 91 (24), 6269-6271.

23. Miyamoto, S.; Kollman, P. A., Settle - an Analytical Version of the Shake and Rattle Algorithm for Rigid Water Models. J. Comput. Chem. 1992, 13 (8), 952-962.

24. Darden, T.; York, D.; Pedersen, L., Particle Mesh Ewald - an N.Log(N) Method for Ewald Sums in Large Systems. J. Chem. Phys. 1993, 98 (12), 10089-10092.

25. Hess, B.; Bekker, H.; Berendsen, H. J. C.; Fraaije, J. G. E. M., LINCS: A linear constraint solver for molecular simulations. J. Comput. Chem. 1997, 18 (12), 1463-1472.

26. Shim, Y.; Kim, H. J., Solvation of Carbon Nanotubes in a Room-Temperature Ionic Liquid. Acs Nano 2009, 3 (7), 1693-1702.

27. Hess, B.; Kutzner, C.; van der Spoel, D.; Lindahl, E., GROMACS 4: Algorithms for Highly Efficient, Load-Balanced, and Scalable Molecular Simulation. J. Chem. Theo. Comp. 2008, 4 (3), 435-447.

28. Humphrey, W.; Dalke, A.; Schulten, K., VMD: Visual molecular dynamics. J. Mol. Graphics 1996, 14 (1), 33-38.

29. Chu, B., Structure and Dynamics of Block-Copolymer Colloids. Langmuir 1995, 11 (2), $414-$ 421.

30. Moore, V. C.; Strano, M. S.; Haroz, E. H.; Hauge, R. H.; Smalley, R. E.; Schmidt, J.; Talmon, Y., Individually suspended single-walled carbon nanotubes in various surfactants. Nano Lett. 2003, 3 (10), 1379-1382. 
31. Granite, M.; Radulescu, A.; Cohen, Y., Small-Angle Neutron Scattering from Aqueous Dispersions of Single-Walled Carbon Nanotubes with Pluronic F127 and Poly (vinylpyrrolidone). Langmuir 2012, 28 (30), 11025-11031.

32. Xin, X.; Xu, G.; Zhao, T.; Zhu, Y.; Shi, X.; Gong, H.; Zhang, Z., Dispersing carbon nanotubes in aqueous solutions by a starlike block copolymer. J. Phys. Chem. C 2008, 112 (42), 16377-16384.

33. Pang, J.; Xu, G.; Tan, Y.; He, F., Water-dispersible carbon nanotubes from a mixture of an ethoxy-modified trisiloxane and pluronic block copolymer F127. Colloid. Polym. Sci. 2010, 288 (18), 1665-1675.

34. Shvartzman-Cohen, R.; Florent, M.; Goldfarb, D.; Szleifer, I.; Yerushalmi-Rozen, R., Aggregation and self-assembly of amphiphilic block copolymers in aqueous dispersions of carbon nanotubes. Langmuir 2008, 24 (9), 4625-4632.

35. Hezaveh, S.; Samanta, S.; De Nicola, A.; Milano, G.; Roccatano, D., Understanding the Interaction of Block Co-Polymers with DMPC Lipid Bilayer using Coarse-Grained Molecular Dynamics Simulations. J. Phys. Chem. B 2012, 116 (49), 14333-14345.

36. De Nicola, A.; Zhao, Y.; Kawakatsu, T.; Roccatano, D.; Milano, G., Hybrid particle-field coarse-grained models for biological phospholipids. J. Chem. Theo. Comput. 2011, 7 (9), 2947-2962.

37. De Nicola, A.; Hezaveh, S.; Zhao, Y.; Kawakatsu, T.; Roccatano, D.; Milano, G., Micellar drug nanocarriers and biomembranes: how do they interact? Phys. Chem. Chem. Phys., 2014, $16,5093-5105$.

38. Sarukhanyan, E.; De Nicola, A.; Roccatano, D.; Kawakatsu, T.; Milano, G., Spontaneous Insertion of Carbon Nanotube Bundles inside Biomembranes: a Hybrid Particle-Field CoarseGrained Molecular Dynamics Study. Phys. Chem. Lett. 2014, 595, 156-166. 
Page 29 of 29

1

2

3

4

5

6

7

8

9 10

11

12

13

14

15

16

17

18

19

20

21

22

23

24

25

26

27

28

29

30

31

32

33

34

35

36

37

38

39

40

41

42

43

44

45

46

47

48

49

50

51

52

53

54

55

56

57

58

59
The Journal of Physical Chemistry

\section{Table of Contents}

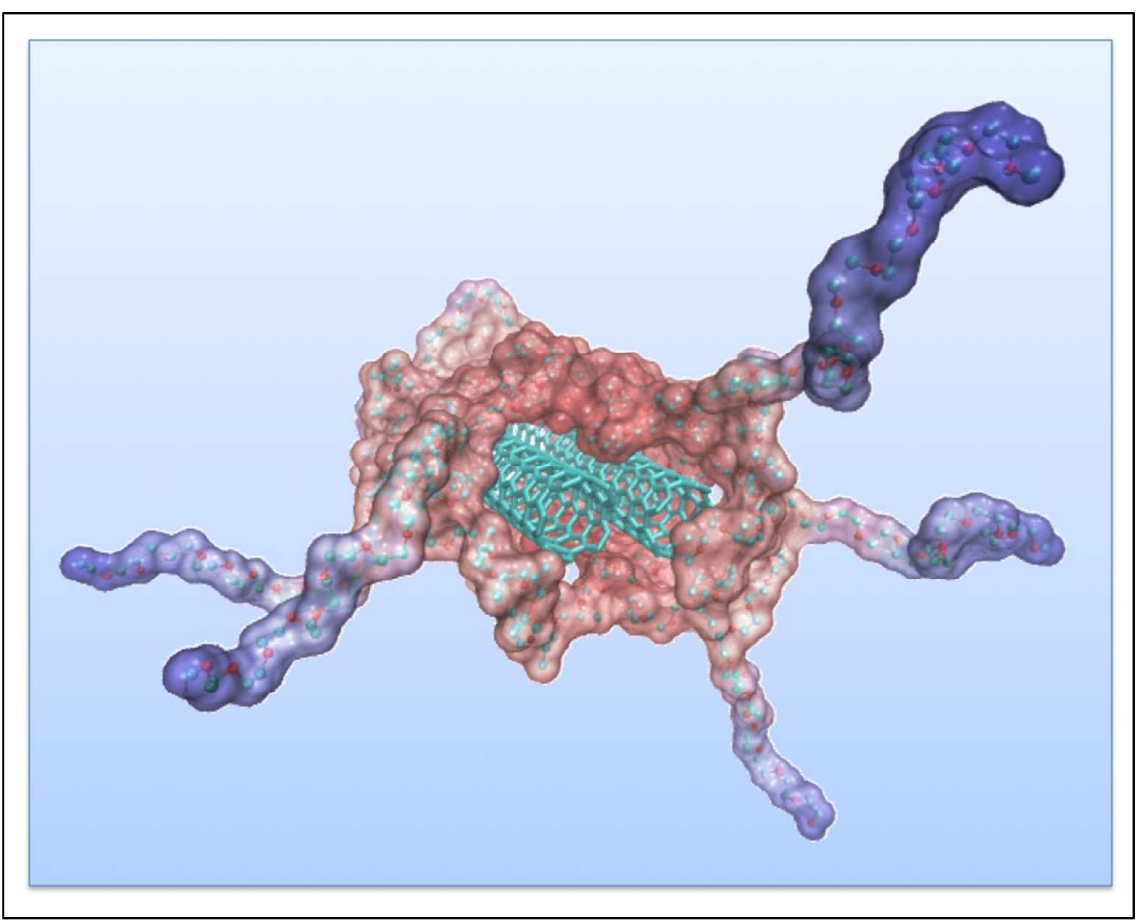

29

ACS Paragon Plus Environment 